Revisão

\title{
Transtorno de estresse pós-traumático: formulação diagnóstica e questões sobre comorbidade
}

\section{Post-traumatic stress disorder: diagnostic formulation and comorbidity issues}

José Waldo S Câmara Filho e Everton B Sougey

Departamento de Neuropsiquiatria da Universidade Federal de Pernambuco. Recife, PE, Brasil

Resumo Objetivos: Atualizar os principais aspectos relacionados ao quadro clínico e ao diagnóstico do transtorno de estresse pós-traumático (TEPT), assim como discutir sua validade como entidade nosológica e sua comorbidade. Métodos: Descrição da sintomatologia clínica, detendo-se em seu significado fenomenológico, e revisão da literatura sobre comorbidade e validação diagnóstica.

Resultados: O TEPT tem sua apresentação clínica dividida em três grupos sintomatológicos relacionados à reexperiência traumática, ao comportamento de esquiva e distanciamento emocional e à hiperexcitabilidade psíquica. A estruturação diagnóstica do TEPT, especialmente a validade do constructo, vem sendo confirmada por evidências crescentes oriundas de estudos de natureza epidemiológica e neurobiológica. A prevalência de transtornos co-mórbidos próxima a $80 \%$ desperta a atenção sobre a maneira em que é conceituado o diagnóstico. Questiona-se se a imprecisão descritiva dos critérios diagnósticos, permitindo a sobreposição de sintomas de outros transtornos, não estaria contribuindo para uma superestimação da prevalência co-mórbida.

Conclusão: O TEPT é considerado válido como diagnóstico, reconhecendo e legitimando a condição clínica, não necessariamente temporária, derivada essencialmente do trauma psicológico.

Descritores Estresse pós-traumático. Transtornos da ansiedade. Diagnóstico.

Abstract Objectives: To update the main aspects of the clinical presentation and diagnosis of post-traumatic stress disorder and to discuss its validity as a nosological entity and its comorbidity.

Methods: It was carried out a description of the clinical symptomatology, focusing on its phenomenological significance and literature review on comorbidity and diagnostic validation.

Results: The clinical manifestations of PTSD can be divided into three symptomatological groups according to traumatic reexperience, avoidance and numbing, and psychic arousal. Growing evidence derived from a number of epidemiological and neurobiological studies has confirmed the PTSD diagnostic structure, especially the construct's validity. The presence of comorbid disorders in about $80 \%$ of the cases draws attention to the diagnosis formulation, whether the diagnostic criteria are imprecise, allowing the superimposition of other disorders' symptoms, and contributing to an overestimation of comorbid prevalence.

Conclusion: PTSD is considered a valid diagnosis, as long as it also recognizes and legitimates the clinical condition, which is not necessarily temporary, derived from the psychological trauma.

Keywords Stress disorders, post-traumatic. Anxiety disorders. Diagnosis.

Trabalho baseado em Dissertação de Mestrado: Transtorno de estresse pós-traumático: características clínicas e sociodemográficas em policiais militares e suas famílias. Universidade Federal de Pernambuco; 1999.

Recebido em 06/11/2000. Revisado em 26/3/2001 e 10/7/2001. Aceito em 8/8/2001.

Fonte de financiamento e conflito de interesses inexistentes. 


\section{Introdução}

Em 1980, a Associação Psiquiátrica Americana publicou a terceira revisão de sua classificação diagnóstica de transtornos mentais, o Manual de Diagnóstico e Estatística dos Distúrbios Mentais (DSM-III). ${ }^{1}$ Nela surgia um novo termo diagnóstico: o transtorno de estresse pós-traumático (TEPT). Os ditos diagnósticos sindrômicos de ocasião, como neurose traumática, síndrome do trauma de estupro, fisioneurose, neurose de compensação e muitos outros, encontraram no TEPT uma forma mais abrangente de conceituação.

Dessa maneira, a validação diagnóstica do TEPT reconheceu o sofrimento de pessoas cuja história traumática não era valorizada como fator determinante de seus padecimentos eram os ansiosos, os deprimidos, os fóbicos ou pior: "os neuróticos, os histéricos, os simuladores". ${ }^{2}$

O TEPT atualmente é muito estudado num movimento que tem sua maior produção nos Estados Unidos, alguns países da Europa, Israel e Oceania. O interesse pelo TEPT evidencia-se pelo número crescente de publicações, pelo surgimento de periódicos exclusivamente dedicados ao tema e pela atividade associativa entre profissionais e pesquisadores. No Brasil, infelizmente, essa entidade clínica ainda é pouco conhecida, raramente diagnosticada e facilmente confundida; são escassas as publicações científicas, e o tema é pouco tratado em eventos especializados.

Não se trata, porém, de transtorno de ocorrência rara: Kessler et al, ${ }^{3}$ por dados obtidos a partir do National Comorbidity Survey (NCS) - extenso estudo epidemiológico americano -, utilizando os critérios do DSM-III-R, estimaram a prevalência do TEPT em 7,8\% da população geral, distribuídos em 5,0\% dos homens e $10,4 \%$ das mulheres.

Dessa forma, o objetivo do presente trabalho é contribuir para o conhecimento do TEPT no Brasil por meio da atualização crítica dos principais aspectos relacionados a sua apresentação clínica e formulação diagnóstica, trazendo também à tona os questionamentos e as respostas disponíveis acerca de sua validade conceitual e comorbidade de outros transtornos psiquiátricos.

\section{Métodos}

A metodologia da presente revisão está inserida dentro de estudo mais amplo. ${ }^{2}$ A recente consolidação da nomenclatura referente aos transtornos pós-traumáticos justificou a ausência de um corte temporal definida na seleção de trabalhos e ensejou a consulta a textos clássicos na descrição de quadros clínicos semelhantes, mas, à época, definidos em diferentes terminologias. Foram utilizados os bancos de dados Medline, Lilacs e Pilots (instrumento de pesquisa bibliográfica eletrônica vinculado ao National Center of PostTraumatic Stress Disorder - principal centro de pesquisas americano sobre o assunto).

\section{Trauma}

Trauma, fundamental na concepção do TEPT, é definido como uma situação experimentada, testemunhada ou confrontada pelo indivíduo, na qual houve ameaça à vida ou à integridade física de si próprio ou de pessoas a ele afetivamente ligadas. Seriam situações essencialmente violentas, como acidentes naturais (p. ex: enchentes, incêndios, soterramentos), acidentes automobilísticos, assaltos, seqüestros, estupros, entre outros. Apesar do caráter objetivo de sua descrição e critérios, sua identificação é muitas vezes difícil, e uma anamnese dirigida pode ser necessária. ${ }^{4}$

\section{Quadro clínico}

Tradicionalmente a sintomatologia do TEPT é organizada em três grandes grupos: o relacionado à reexperiência traumática, à esquiva e distanciamento emocional e à hiperexcitabilidade psíquica.

\section{Reexperiência traumática}

Mesmo estando o perigo afastado e confinado ao passado, o indivíduo pós-traumatizado continuamente revive o ocorrido, vivenciando-o como experiência contemporânea em vez aceitálo como algo pertencente ao passado: "Incapaz de retomar o curso de sua vida porquanto o trauma constantemente está a interrompê-la: é como se o tempo parasse no momento do trauma".

Quase sempre rumina pensamentos de forma improdutiva sobre temas relacionados ao trauma. Estão presentes as lembranças intrusivas e recorrentes: recordações que assaltam o paciente continuamente. São lembranças fixas, que não se alteram com o tempo, de uma nitidez e vividez distintas, além de carregadas de forte componente afetivo e emocional: a lembrança trazendo angústia e sofrimento intensos. Espontâneas, involuntárias, ao surgirem não são facilmente interrompidas, parecendo "ter vida própria". 6

Muitas vezes as lembranças estão fragmentadas em forma de imagens, sons, odores, sensações físicas (náuseas, tonturas e outras) ou emoções (medo, pavor, horror) não conectadas umas às outras por força do que foi denominada dissociação primária. ${ }^{7}$ Com a elaboração psíquica da experiência, essas recordações, que muitas vezes estão guardadas em fragmentos sensoriais com pouco ou nenhum componente de linguagem, vão se integrando e chegando, com o correr do tempo, a se constituir em narrativa que conseguiria traduzir o ocorrido. ${ }^{8}$

Pequenos e mesmo insignificantes estímulos conseguem reavivar as memórias que retornam com toda a força, intensidade e nitidez do acontecimento original. Tais lembranças são freqüentemente detonadas por estímulos relacionados ao evento traumático, desde os mais específicos aos mais genéricos (chuva, trovões, telefone, noticiários e outros). Assim, mesmo um ambiente protegido poderia se tornar perigoso, porquanto a pessoa nunca estaria suficientemente segura de não vir a se deparar com estímulos evocadores.

A relação entre o estado de excitação fisiológica central e a revivescência traumática é estreita: a presença de hiperatividade autonômica que acompanha os estados ansiosos comumente provoca o reaparecimento dos sintomas intrusivos. ${ }^{9}$ Estudos neurobiológicos confirmam essa observação clínica, como nos experimentos em que a administração de substâncias ansiogênicas desencadeia flashbacks em indivíduos portadores do TEPT. ${ }^{10}$ 
A reexperiência também pode ocorrer sob a forma de sonhos aflitivos e pesadelos. Em alguns pacientes, aparece quase diariamente, o que leva inclusive ao temor de dormir.

$\mathrm{O}$ revivescimento ou flashback seria um dos sintomas mais distintivos do TEPT, apesar de pouco freqüente: a vítima como que se "transportaria" à situação traumática, revivendo-a como se ela estivesse acontecendo naquele momento. Alucinações visuais ou auditivas, despersonalização, desrealização ocorreriam durante esses períodos, sugerindo a natureza dissociativa do fenômeno. Alguns apresentam acurada descrição da situação traumática vivida, enquanto outros têm uma qualidade distorcida ou irreal como um sonho. ${ }^{11}$ Vivência semelhante é a da sensação de que o evento está para ocorrer ou está acontecendo novamente, com perda relativa da distinção entre o presente e o passado. Como no soldado cujo trauma principal envolvia a participação numa rebelião de presos e que, ao se deparar com aglomerados de pessoas, freqüentemente não só revia a cena dos amotinados correndo em fuga como se preparava para combatê-los. ${ }^{2}$

Um fenômeno curioso e paradoxal que ocorre em alguns pacientes com TEPT é o da reexposição compulsiva a novos eventos potencialmente traumáticos. Trata-se de uma outra forma particular da revivescência traumática, que não envolve primariamente os processos do pensamento, da lembrança ou do sonho, mas os da ação, do reexperimentar pelo comportamento que se dirige $\mathrm{a}$ - inconscientemente ou não, mas quase sempre sem se aperceber de tal - vivenciar situações que possam se assemelhar ao evento inicial. Assim, na criança, há os jogos repetitivos (normalmente monótonos e sombrios, sem a vivacidade e a leveza das brincadeiras normais que não se modificam com o tempo e demoram a ser interrompidas) que evocam, às vezes de modo quase literal, o trauma. Observam-se adolescentes traumatizados envolvidos em comportamento de risco, seja numa vida sexual promíscua, no uso de drogas de adição ou na delinqüência. No adulto, pode-se refletir na escolha de um companheiro que venha a repetir no casamento atitudes de violência e abuso sofridos anteriormente na casa dos pais, ou no soldado que, ao se reformar, procura atividades em que possa vir a se expor novamente a situações de perigo.

\section{Esquiva e distanciamento emocional}

Os sintomas relativos à repetição da experiência traumática são acompanhados de considerável sofrimento, que a maioria das vítimas busca evitar, afastando-se de qualquer estímulo que possa desencadear o ciclo das lembranças traumáticas.

Aparece, então, a esquiva ativa de pensamentos, sentimentos, conversas, situações e atividades associadas ao trauma como um mecanismo de defesa contra a ansiedade gerada pelo fenômeno intrusivo. ${ }^{9}$

Dessa maneira, por provocar tamanha angústia, o indivíduo pós-traumatizado não economiza esforços no sentido de afastarse dela. As estratégias de esquiva podem ser óbvias ou sutis, relativamente adaptativas ou manifestamente inadequadas que vão da recusa em falar sobre o trauma, ao uso de bebidas alcoólicas ou drogas para obscurecer as memórias, até ao engajamento excessivo e compulsivo em atividades como trabalho, jogo, sexo, entre outras. Verificou-se, em estudo anterior, ${ }^{2}$ que, em uma população policial militar, o comportamento de esquiva relacionava-se à atividade profissional em si: recusa a portar armas, dirigir automóveis, trabalhar em presídios e até vestir a farda foram observadas.

Em alguns casos, acontece da lembrança simplesmente ser esquecida (amnésia psicogênica ou seletiva). É sintoma de natureza dissociativa, evidenciado por lacunas presentes na história relembrada e contada.

Assombrado pela experiência traumática, o paciente tende a reorganizar sua vida para evitar as emoções negativas que as lembranças intrusivas carregam consigo. Dessa maneira, encontra-se o indivíduo com a constante preocupação de se defender da angústia incomparável - tudo o mais se tornando acessório e pouco importante. Essa maior atenção aos estímulos ligados ao trauma leva a um menor envolvimento com situações potencialmente prazerosas e recompensadoras, contribuindo, assim, para a gradativa centralização do trauma na vida do indivíduo. Advém, então, a diminuição do interesse e da participação em atividades sociais significativas, dado que sua energia psíquica está direcionada à evitação de lembranças e a sentimentos relacionados ao trauma.

Se os sintomas intrusivos tentam gradativamente invadir o campo da consciência, o comportamento de esquiva, paradoxalmente, torna-os mais fortes, porquanto adquirem caráter de verdadeira fobia.

O fantasiar - ou o pensar experimentalmente - é algo percebido como potencialmente perigoso, posto haver o medo de que possa vir a quebrar a frágil barreira erguida para afastar as lembranças dolorosas.

Os escritos autobiográficos de Primo Levi ${ }^{12}$ descrevem com precisão tal estado: "No Campo (de extermínio), pensar não serve para nada, porque os fatos acontecem, em geral, de maneira incompreensível; pensar é, também, um mal, porque conserva viva uma sensibilidade que é fonte de dor, enquanto uma clemente lei natural embota essa sensibilidade quando o sofrimento passa de certo limite".

Dessa forma, a vida organiza-se no esforço do "não pensar", "não sentir" e "não planejar", levando a um comportamento não reflexivo, mas impulsivo. Restringe-se a expressão afetiva de um modo geral, que fica assim como que entorpecida e, no dizer de uma paciente, "com os sentimentos anestesiados". ${ }^{2}$ São sintomas de ordem mais grave (numbing symptoms), prediriam cronicidade ${ }^{13} \mathrm{e}$, segundo Foa et al, ${ }^{14}$ os mais distintivos do TEPT.

\section{Hiperexcitabilidade psíquica}

$\mathrm{O}$ terceiro grupo diz respeito àqueles sintomas que representam um estado de hiperexcitabilidade do sistema nervoso central e autonômico e que não se resumiriam apenas a condicionamentos a estímulos traumatogênicos, mas antes seriam reflexos de uma excitação físiológica extrema em resposta a uma variedade ampla de estímulos.

Trata-se dos sintomas mais comuns e também dos menos específicos: especialmente quando em contato com estímulostrauma afins, uma variedade de reações acompanha o humor 
ansioso, como taquicardia, respiração curta ou suspirosa, constrição precordial, "formigamentos", parestesias, sudorese, extremidades frias ou também cefaléias, tonturas, sensação de "oco na cabeça", "peso no estômago", entre outras.

Há a insônia, e aparecem a irritabilidade e a explosividade: sempre alertas, os pacientes passam do estímulo à ação sem o tempo para a necessária reflexão e avaliação criteriosa do estímulo provocador.

A hipervigilância é encontrada comumente: estão sempre em guarda, esperam o pior e reagem como se estivessem sob contínua ameaça de aniquilação. Há a generalização do medo de um mundo percebido como lugar inseguro e imprevisível. A hipervigilância se evidencia no comportamento de quem está constantemente avaliando o ambiente: o indivíduo que, após ser assaltado, continuamente fica a olhar sobre os ombros, a espreitar o ambiente de um lado a outro à procura de sinais que possam se revelar ameaçadores.

Outro sintoma característico é o da resposta de sobressalto exagerada (resposta musculoesquelética súbita, que varia da simples contração de grupos musculares até o assumir de posturas de defesa em resposta a estímulos inócuos: normalmente barulho ou som alto). Os pacientes assustam-se facilmente. Tais sintomas têm correlação neurobiológica interessante, traduzem uma dificuldade na avaliação discriminatória do estímulo e uma incapacidade de modular a resposta, no mais das vezes, extrema e estereotipada. ${ }^{15,16}$

\section{Diagnóstico}

A evolução na maneira de contextualizar a relação trauma e doença pode ser acompanhada à luz de como os principais sistemas de classificação diagnóstica nomeavam os diferentes transtornos. A Tabela descreve, dentro da Classificação Internacional de Doenças (CID) e do DSM americano, a nomenclatura relacionada aos transtornos pós-traumáticos nas diferentes versões ao longo dos anos.
Nota-se que, antes da DSM-III ${ }^{1}$ e da CID- $10,{ }^{17}$ atribuíam-se às reações pós-traumáticas características de impacto agudo e essencialmente transitório. A persistência ou a cronicidade de sintomas implicava uma necessária reconsideração do diagnóstico inicial, já que residiriam em predisposições constitucionais ou psicodinâmicas do indivíduo as causas de uma perturbação que não se resolvesse num breve e preestabelecido período de tempo. O papel do trauma nesse distúrbio, agora prolongado, seria reinterpretado como o de um elemento apenas desencadeador.

Nesse sentido, a CID-10 ${ }^{17}$ é também mais inovadora quando reconhece o poder do evento traumático em provocar, inclusive, modificações duradouras de personalidade (F62.0).

Quanto à classificação norte-americana, a estruturação diagnóstica inicial do TEPT, publicada no DSM-III, ${ }^{1}$ encontrou respaldo nos estudos clínicos e epidemiológicos que se seguiram. Daí que, em relação aos critérios diagnósticos, de sua introdução até a definição atual do DSM-IV, ${ }^{18}$ poucas modificações foram efetuadas ${ }^{19}$ - a maioria delas voltada à definiç̧ão do evento traumático.

Uma outra constatação é a incorporação da palavra estresse na nomenclatura psiquiátrica, apesar de ser conceito oriundo da fisiologia, não se tratar de termo da psicopatologia e de não encontrar consenso em seu entendimento, estando mesmo sujeito a diversas interpretações (embora seja considerado válido como conceito). ${ }^{20}$ Interroga-se se sua utilização não desempenhou um papel na rápida popularização do TEPT nos países do hemisfério Norte. Até porque se observa o uso leigo da palavra estresse como eufemismo - é talvez mais aceitável socialmente dizer que se está "estressado" que propriamente "ansioso" ou "deprimido".

Horowitz, Weiss \& Marmar $^{21}$ resumem as questões relativas ao diagnóstico do TEPT ao apontar a necessidade dos critérios diagnósticos de atender aos quatro aspectos inerentes à validação conceitual de uma categoria diagnóstica:

Tabela - Nomenclatura diagnóstica para transtornos ligados ao estresse traumático nas classificações internacional* e americana

Classificação internacional Classificação americana

CID-6 (1948)/CID-7 ( 1955 )

Desajuste situacional agudo 326.3

CID-8 (1968)

Transtornos transitórios de inadaptação a situações especiais 307

CID-9 (1977)

Reação aguda ao estresse 308

Distúrbios predominantes das emoções 308.0/5

Distúrbios predominantes da consciência 308.1/3

Distúrbio predominantemente psicomotor 308.2/1

Outras $308.3 / 0$

Mista 308.4/8

Não-especificada 308.9/9

CID-10 ( 1992 )

Reação aguda a estresse F43.0

Transtorno de estresse pós-traumático F43.1

Outras reações a estresse grave F43.1

Reação a estresse grave não especificada F43.9

Alteração permanente de personalidade após experiência catastrófica F62.0
Classificação americana

DSM-I (1952)

Distúrbio situacional transitório de personalidade

Reação maciça ao estresse

Reação situacional do adulto

Reação de ajustamento

DSM-II (1968)

Reação de ajustamento da idade adulta 307.3

DSM-III (1980)/DSM-III-R (1987)

Transtorno de estresse pós-traumático 309.89

* O capítulo referente aos transtornos mentais na classificação internacional fora introduzido a partir da CID-6.

DSM-IV ( 1994 )

Transtorno de estresse agudo 308.3

Transtorno de estresse pós-traumático 309.81 
- validade de face: a descrição do transtorno correspondendo acuradamente às experiências e ao comportamento do paciente;

- validade descritiva: o transtorno é descrito de tal forma que permite ser bem diferenciado de outras patologias. Talvez aqui resida a maior crítica à validade do TEPT como entidade diagnóstica, em função do elevado número de associações co-mórbidas (que como se verá adiante pode vir a ser conseqüência da sobreposição de sintomas presentes na descrição de diversas patologias);

- validade preditora: implica que o indivíduo, ao preencher os critérios do transtorno, venha a apresentar uma evolução dentro do esperado curso da doença e venha a responder de determinada maneira ao tratamento instituído;

- validade do constructo: requer algum tipo de evidência que venha a apoiar os modelos teóricos que explicam a formação dos sinais e sintomas do transtorno. Tais evidências existem em grande número, principalmente a partir de estudos neurobiológicos que vêm apontando o TEPT como transtorno de características únicas, já que demonstram a presença de modificações biológicas associadas a sintomas que não são encontrados em outras transtornos, nem são o resultado da exposição a traumas somente. ${ }^{22-24}$ Especialmente intrigantes são aquelas relacionados ao eixo neurohormonal hipotálamo-pituitária-adrenal. ${ }^{24}$

O diagnóstico do TEPT, 20 anos após sua introdução nos sistemas de classificação, é considerado válido com o embasamento vindo de diversas áreas do conhecimento, especialmente de estudos epidemiológicos que têm consistentemente demonstrado a ocorrência do TEPT no universo da população geral e em uma variedade de amostras de populações específicas e das modificações neuroquímicas, hormonais e fisiológicas encontradas nesses pacientes. ${ }^{22,23}$ Há a contribuição de toda uma bateria de instrumentos de avaliação que aumentam a acurácia diagnóstica ${ }^{25,26}$ e dos modelos teóricos que buscam sintetizar os dados de informação adquiridos..$^{27-29}$

\section{Comorbidade}

As questões envolvendo a comorbidade e o TEPT revestemse de grande importância, não só em função de desafiar a validade do diagnóstico mas também pelas implicações etiofisiopatogênicas e as relacionadas a seu curso e tratamento, posto que a presença de outro transtorno psiquiátrico afetaria a apresentação clínica e sua evolução natural. ${ }^{30}$

A coexistência de transtornos psiquiátricos com o TEPT é intrigantemente alta. Friedman \& Yehuda ${ }^{24}$ chegam a afirmar que se um indivíduo preenche os critérios diagnósticos para o TEPT provavelmente também os preencherá para outros transtornos.

Helzer, Robins \& McEvoy, ${ }^{31}$ dentro do Epidemiologic Catchment Survey, outro estudo nacional epidemiológico americano, encontraram prevalência co-mórbida em $80 \%$. Os portadores de TEPT teriam probabilidade duas vezes maior de apresentar também outro transtorno e maior risco para TOC, distimia, transtorno maníaco-depressivo (aqui incluídos o episódio depressivo maior e/ou maníaco) e abuso de substâncias psicoativas.
Kessler et al, ${ }^{3}$ por meio de dados do NCS, encontraram prevalência co-mórbida de pelo menos um outro transtorno em $88,3 \%$ dos homens e $79 \%$ das mulheres, sendo mais comuns alcoolismo e abuso de outras substâncias psicoativas, depressão e distimia, transtornos de conduta, transtornos de ansiedade (TAG, transtorno de pânico, fobias) e mania.

Em amostra de policiais militares atendidos em ambulatório, Câmara Filho ${ }^{2}$ encontrou a presença de transtorno co-mórbido em 53,3\% dos pacientes com TEPT, destacando a depressão e o alcoolismo.

Dentre as patologias co-mórbidas mais freqüentes se destacam:

- transtornos de humor, especialmente depressão em $46 \%$ a $51 \% ; 3,32,33$

- transtornos de ansiedade em $29 \%$ a $56,1 \% ; 3,32,33$

- abuso/dependência de substâncias psicoativas em $27 \%$ a $80 \% ; 3,34$

- transtorno obsessivo-compulsivo em $13 \%,{ }^{32}$ risco dez vezes maior de apresentá-lo; ${ }^{31}$

- transtornos somatoformes: pacientes com TEPT teriam probabilidade três vezes maior de apresentar sintomas de somatização, ${ }^{35}$

- transtornos dissociativos: Saxe et $\mathrm{al}^{36}$ encontraram TEPT em $90 \%$ dos pacientes com quadros dissociativos internados em hospital psiquiátrico;

- transtornos de personalidade: Southwick, Yehuda \& Giller Jr. ${ }^{37}$ estudando população de veteranos de guerra, encontraram com maior freqüência transtornos de personalidade borderline, obsessivo-compulsivo e paranóide. Lauterbach \& Vrana, ${ }^{38} \mathrm{em}$ trabalho recente com estudantes universitários, apontam prevalência maior de transtornos de personalidade tipo borderline, narcisista, paranóide, passivo-agressivo e esquizotípico. Sugerem que a maior freqüência em transtornos de personalidade do grupo A (esquizotípico e paranóide) poderia ser explicada pela dificuldade dessas pessoas de obter suporte social e familiar que, sabidamente, diminuem o impacto traumático e aceleram a recuperação.

Há que ser feito comentário sobre a validez metodológica dos estudos de comorbidade: sua heterogeneidade não permite a síntese desejável de resultados, posto haver entre eles diferenças marcantes, sejam na definição do evento traumático (critérios distintos baseados nas diferentes DSM e CID-10), nos instrumentos de avaliação empregados, na população abrangida, no corte retrospectivo ou prospectivo (raros ainda), entre outros.

Não obstante, a prevalência encontrada permanece consistentemente alta, o que levanta uma série de pertinentes dúvidas que dizem respeito a questões que vão de sua validade como transtorno psiquiátrico à adequação dos instrumentos de avaliação disponíveis e aos próprios critérios operacionais para o diagnóstico. Indaga-se, por exemplo:

- seriam os transtornos coexistentes condições distintas, de diferentes origens e patogênese?

- estar-se-ia diante de um artefato diagnóstico face à sobreposição de sintomas? Ao se comparar os critérios diagnósticos do TEPT com, por exemplo, os do transtorno de ansiedade generalizada, transtorno de pânico ou 
depressão, nota-se que eles compartilham entre si de vários sintomas, como insônia, diminuição da concentração, irritação, isolamento, desinteresse, pessimismo na depressão e no TEPT (os próprios sintomas relacionados à constrição afetiva e ao distanciamento emocional confundem-se com o humor depressivo), hiperexcitação autonômica, insônia, irritabilidade, reações de sobressalto nos transtornos ansiosos e no TEPT (Figura).

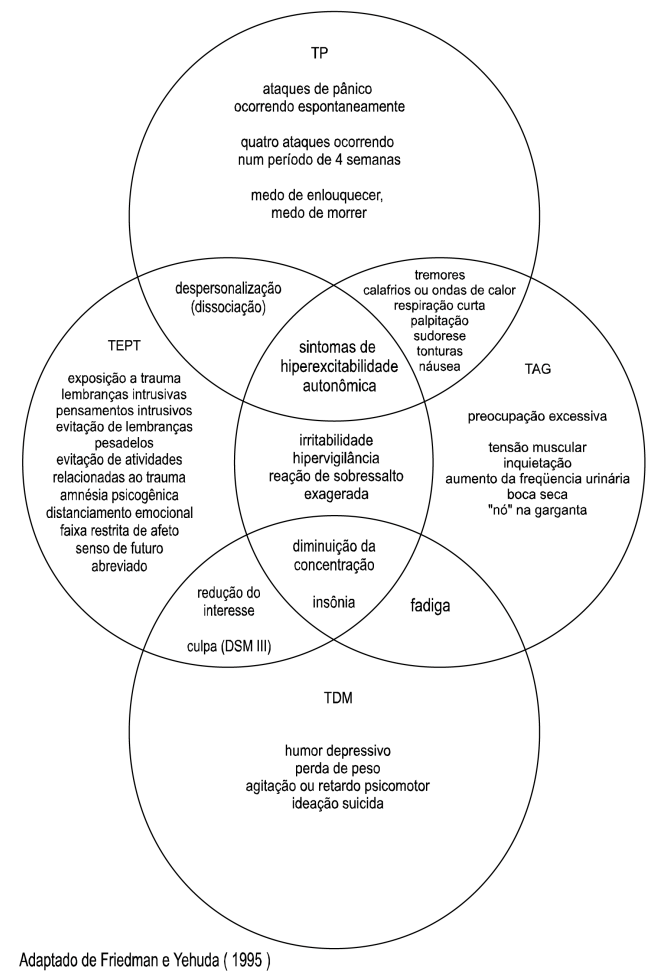

Figura - Sobreposição de sintomas entre transtorno de estresse póstraumático (TEPT), transtorno de pânico (TP), transtorno de ansiedade generalizada (TAG) e transtorno depressivo maior (TDM).

Na presença de um transtorno afetivo ou de ansiedade, provavelmente preencher-se-ão os critérios do DSM-IV correspondentes ao grupo D de sintomas (hiperexcitabilidade psíquica), além do que muitos indivíduos também preencherão os critérios correspondentes ao grupo B (repetição da experiência traumática), visto que são sintomas comuns naqueles que vivenciam experiências traumáticas independentemente de adoecer ou não. Friedman \& Yehuda $^{24}$ questionam se não se poderia levantar a hipótese de, ao se encontrar indivíduo deprimido e portador de TEPT (o achado co-mórbido mais comum), não se estaria de fato com indivíduo portador de TEPT de um subtipo depressivo.

- Tratar-se-ia de diferentes expressões fenomenológicas de patofisiologia única? Variantes de uma síndrome pós-traumática maior? Sabe-se que o estresse e o trauma de uma forma geral estão presentes, senão na gênese, mas no desencadear de várias patologias. ${ }^{22}$ Assim poderia haver duas ou mais síndromes (guardando entre si uma relação de dependência, espécie de submissão hierárquica) cujos destaque, relativa proeminência ou gravidade dependeriam da seqüência de seus desenvolvimentos sintomatológicos. ${ }^{39}$
Destaca-se que a evolução crônica do TEPT (duração superior a dois anos) foi considerada, em estudo anterior dos autores, ${ }^{2}$ fator de risco significativo para o aparecimento de transtorno co-mórbido, corroborando a afirmação de Yehuda \& McFarlane $^{22}$ de que a comorbidade psiquiátrica se desenvolveria no decorrer do tempo, com sua evolução possibilitando o descortinar de uma série de processos psicopatológicos secundários.

- Existiria um viés (bias) de seleção nos estudos de comorbidade? Os pacientes que buscariam mais a assistência médica e psicológica seriam aqueles com outros transtornos associados (os mais graves), contribuindo para uma estimativa de prevalência co-mórbida superestimada.

- Existiria um viés de informação? Davidson \& $\mathrm{Foa}^{40}$ lembram que a maioria dos estudos sobre comorbidade utiliza o Diagnostic Interview Schedule, cujas inerentes limitações (não se sabe em quanto) possam se refletir nos resultados.

São postulações válidas para as quais não se têm ainda respostas definitivas, mas é inegável que a formulação diagnóstica do TEPT permanece imprecisa e, acredita-se, de pouca confiabilidade. Tal imprecisão possibilitaria, assim, a sobreposição de diferentes diagnósticos que apresentam critérios operacionais e descrições clínicas com termos e sintomas de descrição semelhante ao do TEPT, mas que possuem uma compreensão fenomenológica fundamentalmente diferente quando relacionados a uma ou outra entidade nosológica. Não se define, no presente estudo, a abordagem fenomenológica como mero sinônimo para descrição de sinais e sintomas (uso que se degenerou, sendo conceitualmente desinteressante), ${ }^{41}$ mas com o entendimento psicopatológico clássico, jaspersiano, que implica o estudo da real experiência subjetiva do sujeito. Dessa forma, quando se compara o significado da manifestação de determinados sintomas do grupo $\mathrm{C}$ de diagnóstico do TEPT no DSM-IV (esquiva e distanciamento emocional) com alguns sintomas presentes na depressão, percebe-se melhor tal imprecisão descritiva.

Enquanto na depressão a presença de isolamento social e diminuição do interesse e da participação em atividades significativas é tradução de insegurança, baixa auto-estima, sentimento de inferioridade e, principalmente, anedonia ou hipobulia, no TEPT seria a conseqüência da fuga de estímulostrauma afins, de uma postura de esquiva que se generaliza.

Na depressão, a descrição de faixa restrita de afeto seria secundária ao estado de hiporreatividade, inibição e de uma modulação depressiva dos afetos. No TEPT, a retração afetiva aparece como defesa ao estado de hiperexcitação, como tentativa de se proteger de emoções que poderiam exacerbar os sintomas ansiosos relacionados, daí a impressão de distanciamento, frieza e indiferença que o indivíduo transmite.

Para o sentimento de futuro abreviado, na depressão seria fruto do pessimismo, niilismo, sentimento de incapacidade e desmoralização. No TEPT traduziria a visão do futuro como algo comprovadamente incerto, imprevisível, inseguro e perigoso, sensação que suscita a pergunta do porquê investir ou se envolver, já que a ameaça traumática estaria ainda viva na lembrança e no comportamento. 


\section{Conclusão}

A delimitação diagnóstico do TEPT oferecida pelo DSM-III em 1980 possibilitou uma verdadeira "explosão" de estudos a respeito. Investigações com metodologia mais precisa envolvendo diferentes campos de estudo - epidemiologia, nosologia, neurobiologia, terapêutica, entre outros - confirmaram sua validade diagnóstica, e poucas modificações em seus critérios operacionais foram feitas nas diferentes classificações que se sucederam.

Mesmo estabelecida a validade do TEPT como diagnóstico, não o exime, porém, de questionamentos que ainda persistem e envolvem sua classificação em grupos nosológicos, a indagação sobre a elevada prevalência de transtornos co-mórbidos

\section{Referências}

1. American Psychiatric Association. Manual de diagnóstico e estatística dos distúrbios mentais: DSM-III. Lisboa: Portuguesa de Livros Técnicos e Científicos; 1986.

2. Câmara Filho JWS. Transtorno de estresse pós-traumático: características clínicas e sociodemográficas em policiais militares e suas famílias (dissertação). Recife: Universidade Federal de Pernambuco; 1999.

3. Kessler RC, Sonnega A, Bromet E, Hughes M, Nelson CB. Posttraumatic stress disorder in the national comorbidity survey. Arch Gen Psychiatry 1995;52:1048-60.

4. Câmara Filho JWS, Sougey EB. Estupro e transtorno de estresse póstraumático: aspectos epidemiológicos e clínicos. J Bras Psiquiatr 1999; 48:547-53.

5. Herman J. Trauma and recovery. New York: Basic Books; 1997.

6. Weiss DS. Structured clinical interview techniques. In: Wilson JP, Keane TM. Assessing psychological trauma and PTSD. New York: Guilford; 1997. p. 493-511.

7. Kolk BA van der, Hart O van der, Marmar CR. Dissociation and information processing in posttraumatic stress disorder. In: Kolk BA van der, McFarlane AC, Weisaeth L. Traumatic stress: the effects of overwhelming experience on mind, body and society. New York: Guilford; 1996. p. 303-27.

8. Kolk BA van der. Trauma and memory. In: Kolk BA van der, McFarlane AC, Weisaeth L. Traumatic stress: the effects of overwhelming experience on mind, body and society. New York: Guilford; 1996. p. 279-302.

9. McFarlane AC. Avoidance and intrusion in posttraumatic stress disorder. J Nerv Ment Dis 1992;180:439-45.

10. Rainey JM, Aleem A, Ortiz A, Yeragani V, Pohl R, Berchou R. A laboratory procedure for the induction of flashbacks. Am J Psychiatry 1987;144:1317-19.

11. Krystal JH, Bennett A, Bremner JD, Southwick SM, Charney DS. Toward a cognitive neuroscience of dissociation and altered memory functions in post-traumatic stress disorder. In: Friedman MJ, Charney DS, Deutch AY. Neurobiological and clinical consequences of stress. Philadelphia: Lippincott-Raven; 1995. p. 239-69.

12. Levi P. É isto um homem? Rio de Janeiro: Rocco; 1997.

13. Feeny NC, Zoellner LA, Fitzgibbons LA, Foa EB. Exploring the roles of emotional numbing, depression, and dissociation in PTSD. J Traum Stress 2000;13:489-98.

14. Foa EB, Riggs DS, Gershuny BA. Arousal, numbing and intrusion: symptom structure of PTSD following assault. Am J Psychiatry 1995;152:116-20.

15. McFarlane AC, Weber DL, Clark CR. Abnormal stimulus processing in posttraumatic stress disorder. Biol Psychiatry 1993;34:311-20.

16. Shalev AY, Rogel-Fuchs Y. Psychophysiology of the posttraumatic stress disorder: from sulfur fumes to behavioral genetics. Psychosom Med 1993;55:413-23. e as críticas sobre a precisão de seus critérios descritivos.

Espera-se que o refinamento de critérios operacionais para o diagnóstico por instrumentos de avaliação específica, estudos epidemiológicos mais apurados ou até mesmo o desenvolvimento de exames complementares possam vir, no futuro, a dirimir as dúvidas que ainda persistem.

Não há como negar, todavia, que o grande mérito da introdução do TEPT, ao longo de quase um século de debates, está em reconhecer e legitimar a condição clínica essencialmente derivada do trauma psicológico (apesar do componente individual e constitucional, sem o qual nenhuma reação aconteceria), sendo essa condição não intrinsecamente temporária, admitindo-se sua perpetuação e cronicidade.

17. Organização Mundial da Saúde. Classificação de transtornos mentais e de comportamento da CID-10: Descrições clínicas e diretrizes diagnósticas. Porto Alegre: Artes Médicas; 1993.

18. American Psychiatric Association. Manual diagnóstico e estatístico de transtornos mentais: DSM-IV. $4^{\mathrm{a}}$ ed. Porto Alegre: Artes Médicas; 1995.

19. Wilson JP. The historical evolution of PTSD Diagnostic criteria: from Freud to DSM-IV. In: Everly Jr GS, Lating JM. Psychotraumatology: key papers and core concepts in post-traumatic stress. New York: Plenum; 1995. p. 9-26.

20. Goldstein DS. Stress as a scientific idea: a homeostatic theory of stress and distress. Homeostasis 1995;36:177-215.

21. Horowitz MJ, Weiss DS, Marmar C. Diagnosis of posttraumatic stress disorder. J Nerv Ment Dis 1987;175:267-8.

22. Yehuda R, McFarlane AC. Conflict between current knowledge about posttraumatic stress disorder and its original conceptual basis. Am J Psychiatry 1995;152:1705-13.

23. Friedman MJ. Biological approaches to the diagnosis and treatment of post-traumatic stress disorder. In: Everly Jr GS, Lating JM. Psychotraumatology: key papers and core concepts in post-traumatic stress. New York: Plenum; 1995. p. 171-94.

24. Friedman MJ, Yehuda R. Post-Traumatic stress disorder and comorbidity: psychobiological approaches to differential diagnosis. In: Friedman MJ, Charney DS, Deutch AY. Neurobiological and clinical consequences of stress. Philadelphia: Lippincott-Raven; 1995. p. 429-45.

25. Carlson EB. Trauma research methodology. Lutherville (MD): Sidran Press; 1996.

26. Wilson JP, Keane TM. Assessing psychological trauma and PTSD. New York: Guilford; 1997.

27. Horowitz MJ. Stress-response syndromes: a review of posttraumatic and adjustment disorders. Hosp Commun Psychiatry 1986;37:241-9.

28. Silove D. Is posttraumatic stress disorder an overlearned survival response? An evolutionary-learning hypothesis. Psychiatry 1998;61:181-90.

29. Everly Jr GS. An integrative two-factor model of pos-traumatic stress. In: Everly Jr GS, Lating JM. Psychotraumatology: key papers and core concepts in post-traumatic stress. New York: Plenum; 1995. p. 27-48.

30. Brady KT. Posttraumatic stress disorder and comorbidity: recognizing the many faces of PTSD. J Clin Psychiatry 1997;58(Suppl):12-5.

31. McFarlane AC, Papay P. Multiple diagnoses in posttraumatic stress disorder in the victims of a natural disaster. J Nerv Ment Dis 1992;180:498-504.

32. Helzer JE, Robins LN, McEvoy L. Post-traumatic stress disorder in the general population: findings of the epidemiologic catchment area survey. N Engl J Med 1987;317:1630-4.

33. Ferrada-Noli M, Asberg M, Ormstad K, Lundin T, Sundbom E. Suicidal behaviour after severe trauma. Part 1: PTSD diagnosis, psychiatric comorbidity and assesments of suicidal behaviour. J Traum Stress 1998;11:103-12. 
34. Kofoed L, Friedman MJ, Peck R. Alcoholism and drug abuse in patients with PTSD. Psychiatr Q 1993;64:151-71.

35. Andreski P, Chilcoat H, Breslau N. Post-traumatic stress disorder and somatization symptoms: a prospective study. Psychiatry Res 1998; 79:131-8.

36. Saxe GN, Kolk BA van der, Berkowitz R, Chinman G, Hall K, Lieberg $\mathrm{G}$, et al. Dissociative disorders in psychiatric inpatients. Am J Psychiatry 1993;150:1037-42.

37. Southwick SM, Yehuda R, Giller Jr EL. Personality disorders in treatment-seeking combat veterans with posttraumatic stress disorder. Am J Psychiatry 1993;150:1020-3.

38. Lauterbach D, Vrana S. Personality profiles of persons experiencing different numbers and types of trauma. Poster presented at the $14^{\text {th }}$ Annual Conference of the International Society for Traumatic Stress Studies; 1998 Nov 20-23; Washington (DC); 1998.

39. McGorry PD, Chanen A, McCarthy E, Riel R van, McKenzie D, Singh BS. Posttraumatic stress disorder following recent-onset psychosis. J Nerv Ment Dis 1991;179:253-8.
40. Davidson JRT, Foa EB. Diagnostic issues in posttraumatic stress disorder: considerations for the DSM-IV. J Abnormal Psychol 1991;100:346-55.

41. Sims A. Sintomas da mente: introdução à psicopatologia descritiva. Porto Alegre: Artmed; 2001.

Correspondência

José Waldo S Câmara Filho

Real da Torre, 375/802, Madalena

50610-000 Recife, PE, Brasil

E-mail:jwcamara@uol.com.br 